\title{
On the Titchmarsh convolution theorem
}

\author{
Seçil Gergün, lossif V. Ostrovskii and Alexander Ulanovskii
}

\section{Introduction and statement of results}

Let $M$ be the set of all finite complex-valued Borel measures $\mu \neq 0$ on $\mathbf{R}$. Set

$$
l(\mu)=\inf (\operatorname{supp} \mu)
$$

The classical Titchmarsh convolution theorem (see, e.g. [4], Chapter VI.F, [6], $\S 16.2$ ) claims that if measures $\mu_{1}, \mu_{2}, \ldots, \mu_{n}$ belong to $M$ and satisfy

$$
l\left(\mu_{j}\right)>-\infty, \quad j=1,2, \ldots, n,
$$

then

$$
l\left(\mu_{1} * \mu_{2} * \ldots * \mu_{n}\right)=l\left(\mu_{1}\right)+l\left(\mu_{2}\right)+\ldots+l\left(\mu_{n}\right),
$$

where ' $*$ ' denotes the operation of convolution.

Simple examples show that condition (1) is essential. One may set

$$
\mu_{1}=\sum_{m=0}^{\infty} \frac{\delta_{-k m}}{m !}, \quad \mu_{2}=\sum_{m=0}^{\infty}(-1)^{m} \frac{\delta_{-k m}}{m !}, \quad k>0,
$$

where $\delta_{x}$ is the unit measure concentrated at the point $x$. Clearly, $\mu_{1} * \mu_{2}=\delta_{0}$. We see that $l\left(\mu_{1}\right)=l\left(\mu_{2}\right)=-\infty$ while $l\left(\mu_{1} * \mu_{2}\right)=0$.

It was Y. Domar [2] who first established that condition (1) can be replaced by a sufficiently fast decay of $\mu_{j}$ at $-\infty$ : there exists $a>2$, such that

$$
\left|\mu_{j}\right|((-\infty, x))=O\left(\exp \left(-|x|^{a}\right)\right), \quad x \rightarrow-\infty, j=1,2, \ldots, n .
$$

The best possible condition on decay of $\mu_{j}$ was obtained in [8]. 
Theorem A. ([8]) If $\mu_{j} \in M$ and the condition

$$
\left|\mu_{j}\right|((-\infty, x))=O(\exp (-c|x| \log |x|)), x \rightarrow-\infty, \quad \text { for all } c>0,
$$

holds for $j=1,2, \ldots, n$, then (2) remains true.

One can easily check that the measures $\mu_{j}$ in example (3) satisfy (4) in which 'for all $c>0$ ' is replaced by ' $c=1 / k$ '. Hence, condition (4) in Theorem A is sharp.

It turns out that there is a close connection between Theorem $\mathrm{A}$ and a certain 'quasi-analytic' property of the second convolutions of measures.

Theorem B. ([8]) Suppose $\nu_{1}, \nu_{2} \in M$ and satisfy (4). If $l\left(\nu_{1}\right)=-\infty$ and $\left.\nu_{1}^{2 *}\right|_{(-\infty, a)}=\left.\nu_{2}^{2 *}\right|_{(-\infty, a)}$ for some $a \in \mathbf{R}$, then $\nu_{1}^{2 *} \equiv \nu_{2}^{2 *}$.

Thus, every measure $\nu \in M$ satisfying the assumptions of Theorem A has the property that the second convolution $\nu^{2 *}$ is uniquely determined by its values on any fixed half-line $(-\infty, a)$. For proof, it suffices to apply Theorem $\mathrm{A}$ to the measures $\mu_{1}=\nu_{1}+\nu_{2}$ and $\mu_{2}=\nu_{1}-\nu_{2}$.

In fact, all $n$-fold convolutions $\nu^{n *}$ have a similar property. Moreover, if $n \geq 3$ then restriction (4) can be substantially weakened.

Theorem C. ([8]) Suppose $n \geq 3, \nu_{1}, \nu_{2} \in M$ and satisfy the condition

$$
\left|\nu_{j}\right|((-\infty, x))=O(\exp (-c|x|)), x \rightarrow-\infty, \text { for all } c>0, j=1,2
$$

If $l\left(\nu_{1}\right)=-\infty$ and $\left.\nu_{1}^{n *}\right|_{(-\infty, a)}=\left.\nu_{2}^{n *}\right|_{(-\infty, a)}$ for some $a \in \mathbf{R}$, then $\nu_{1}^{n *} \equiv \nu_{2}^{n *}$.

Restrictions (4) and (5) in Theorems B and C are sharp (see [8]).

Observe that

$$
\nu_{1}^{n *}-\nu_{2}^{n *}=\left(\nu_{1}-\nu_{2}\right) *\left(\nu_{1}-e^{2 \pi i / n} \nu_{2}\right) * \ldots *\left(\nu_{1}-e^{2 \pi i(n-1) / n} \nu_{2}\right) .
$$

Hence, if $n \geq 3$, the difference $\nu_{1}^{n *}-\nu_{2}^{n *}$ can be represented as the convolution of linearly dependent measures. One may ask if there is an extension of Theorem A to linearly dependent measures in which restriction (4) is replaced with a weaker restriction (5). It is established in this paper that Theorem $\mathrm{C}$ can be easily deduced from a general result which extends Theorem A to linearly dependent measures. This result will be formulated and proved in Section 5 . In our first result we extend Theorem A to measures connected by a linear equation. 
Theorem 1. If $\mu_{1}, \mu_{2}, \ldots, \mu_{n-1} \in M, n \geq 3$, are linearly independent over $\mathbf{C}$, satisfy (5) and

$$
\mu_{n}=\mu_{1}+\mu_{2}+\ldots+\mu_{n-1}
$$

then (2) remains true.

Condition (5) is sharp: the statement ceases to be true if 'for all' in (5) is replaced with 'there exists'.

We derive this theorem from the following factorization theorem in the class $H^{\infty}\left(\mathbf{C}_{+}\right)$of functions analytic and bounded in the upper half-plane $\mathbf{C}_{+}$.

Theorem 2. Let $h \neq 0$ belong to $H^{\infty}\left(\mathbf{C}_{+}\right)$. Suppose that $h=g_{1} g_{2} \ldots g_{n}$ where the functions $g_{j}, j=1,2, \ldots, n, n \geq 3$, are analytic in $\mathbf{C}_{+}$and satisfy the conditions:

(i) there exists $H>0$ such that $\sup \left\{\sum_{j=1}^{n}\left|g_{j}(z)\right|: 0<\operatorname{Im} z<H\right\}<\infty$;

(ii) $g_{1}, g_{2}, \ldots, g_{n-1}$ are linearly independent over $\mathbf{C}$ and

$$
g_{n}=g_{1}+g_{2}+\ldots+g_{n-1} \text {. }
$$

Then there exist constants $b_{j} \in \mathbf{R}$ such that

$$
g_{j}(z) \exp \left(i b_{j} z\right) \in H^{\infty}\left(\mathbf{C}_{+}\right), \quad j=1,2, \ldots, n .
$$

The proof of Theorem 2 is based on two results. The first result is the following immediate corollary of H. Cartan's second main theorem for analytic curves.

Theorem D. ([1]) Let $f_{1}, f_{2}, \ldots, f_{n}, n \geq 3$, be functions analytic in the unit disc whose zeros satisfy the Blaschke condition. If $f_{1}, f_{2}, \ldots, f_{n-1}$ are linearly independent over $\mathbf{C}$ and $f_{n}=f_{1}+f_{2}+\ldots+f_{n-1}$, then

$$
T\left(r, \frac{f_{j}}{f_{n}}\right)=O\left(\log \frac{1}{1-r}\right), \quad r \rightarrow 1, j=1, \ldots, n-1,
$$

where $T$ denotes the Nevanlinna characteristic.

The second result is the following theorem on representation for functions harmonic in $\mathbf{C}_{+}$which we believe is of independent interest.

Theorem 3. Let $u$ be a real-valued function harmonic in $\mathbf{C}_{+}$which satisfies the conditions:

(i) there exists a sequence $\left\{r_{k}\right\}_{k=1}^{\infty}, r_{k} \rightarrow \infty$, such that

$$
\int_{0}^{\pi} u^{+}\left(r e^{i \varphi}\right) \sin \varphi d \varphi \leq \exp (o(r)), \quad r=r_{k} \rightarrow \infty
$$

(ii) there exists $H>0$, such that

$$
\sup _{0<y<H} \int_{-\infty}^{\infty} \frac{|u(x+i y)|}{1+x^{2}} d x<\infty
$$


Then $u$ admits the representation

$$
u(z)=\frac{y}{\pi} \int_{-\infty}^{\infty} \frac{d \nu(t)}{(t-x)^{2}+y^{2}}+k y, \quad z=x+i y \in \mathbf{C}_{+},
$$

where $k \in \mathbf{R}$ is a constant and $\nu$ is a real-valued Borel measure on $\mathbf{R}$ such that

$$
\int_{-\infty}^{\infty} \frac{d|\nu|(t)}{1+t^{2}}<\infty
$$

Theorem 3 differs from the known results ([6], p. 109, [5], p. 233) since it contains a much weaker assumption (7) on the growth of $u^{+}$for representation (9) to hold. For instance, in [5], p. 233, one assumes that $u^{+}(z)=O(|z|),|z| \rightarrow \infty$.

The rest of the paper is organized as follows. In Section 2 we prove Theorem 3 after two preliminary results (Lemmas 2.1 and 2.2). These lemmas may be of interest on their own. Section 3 contains the proof of Theorem 2, which can be viewed as the main part of the proof of Theorem 1. In Section 4, we derive Theorem 1 from Theorem 2. Finally, Section 5 contains a generalization of Theorem 1 to the case when the dimension of the linear $\operatorname{span}$ of $\mu_{1}, \ldots, \mu_{n}$ is less than $n-1$.

\section{Proof of Theorem 3}

Lemma 2.1. Let $u$ be a (complex-valued) function harmonic in $\mathbf{C}_{+}$and satisfying

$$
\sup _{0<s<H} \int_{-\infty}^{\infty} \frac{|u(t+i s)|}{1+t^{2}} d t<\infty \quad \text { for some } H>0
$$

Then $u$ admits the representation

$$
u(z)=\frac{y}{\pi} \int_{-\infty}^{\infty} \frac{d \nu(t)}{(x-t)^{2}+y^{2}}+U(z), \quad z=x+i y \in \mathbf{C}_{+}
$$

where $\nu$ is a Borel measure on $\mathbf{R}$ satisfying (10) and $U$ is a function harmonic in the whole plane $\mathbf{C}$ such that $U(x)=0, x \in \mathbf{R}$.

Proof. Consider the family of Borel measures on $\mathbf{R}$,

$$
\sigma_{s}(E)=\int_{E} \frac{u(t+i s)}{1+t^{2}} d t, \quad 0<s<H
$$


By (11), each sequence $\left\{\sigma_{s_{k}}\right\}_{k=1}^{\infty}, \lim _{k \rightarrow \infty} s_{k}=0$, contains a subsequence (which we also denote by $\left\{\sigma_{s_{k}}\right\}_{k=1}^{\infty}$ ) weak-star convergent to a finite Borel measure $\sigma$ on $\mathbf{R} \cup\{\infty\}$. Hence

$$
\lim _{k \rightarrow \infty} \frac{y}{\pi} \int_{-\infty}^{\infty} \frac{u\left(t+i s_{k}\right)}{(x-t)^{2}+y^{2}} d t=\frac{y}{\pi} \int_{-\infty}^{\infty} \frac{1+t^{2}}{(x-t)^{2}+y^{2}} d \sigma(t)+\frac{y}{\pi} \sigma(\{\infty\}) .
$$

Consider the family of functions

$$
U_{s}(z)=u(z+i s)-\frac{y}{\pi} \int_{-\infty}^{\infty} \frac{u(t+i s)}{(x-t)^{2}+y^{2}} d t, \quad z=x+i y \in \mathbf{C}_{+}, 0<s<\frac{1}{2} H .
$$

Every function $U_{s}$ is harmonic in $\mathbf{C}_{+}$, continuous in $\overline{\mathbf{C}}_{+}$and $U_{s}(x)=0$ for $x \in \mathbf{R}$. By the symmetry principle, it can be harmonically extended to $\mathbf{C}$ and $U_{s}(z)=-U_{s}(\bar{z})$, $z \in \mathbf{C}$.

The family $\left\{U_{s}: 0<s<\frac{1}{2} H\right\}$ is uniformly bounded in any rectangle $\Pi_{R, H}=\{z$ : $\left.|\operatorname{Re} z| \leq R,|\operatorname{Im} z| \leq \frac{1}{4} H\right\}$. Indeed, (14) and (11) imply that there exists $C>0$ such that

$$
\int_{-\infty}^{\infty} \frac{\left|U_{s}(x+i y)\right|}{1+x^{2}} d x \leq C \text { for }|y| \leq \frac{1}{2} H, 0<s<\frac{1}{2} H
$$

Whence we obtain, for $z \in \Pi_{R, H}, \varrho=\frac{1}{4} H, \zeta=\xi+i \eta$,

$$
\begin{aligned}
\left|U_{s}(z)\right| & \leq \frac{1}{\pi \varrho^{2}} \iint_{|\zeta-z| \leq \varrho}\left(1+\xi^{2}\right) \frac{\left|U_{s}(\zeta)\right|}{1+\xi^{2}} d \xi d \eta \\
& \leq \frac{1+(|\operatorname{Re} z|+\varrho)^{2}}{\pi \varrho^{2}} \int_{-H / 2}^{H / 2} \int_{-\infty}^{\infty} \frac{\left|U_{s}(\xi+i \eta)\right|}{1+\xi^{2}} d \xi d \eta \leq \frac{1+(R+\varrho)^{2}}{\pi \varrho^{2}} H C .
\end{aligned}
$$

Let $\left\{s_{k}\right\}_{k=1}^{\infty}$ be a sequence such that (13) holds. By the well-known compactness principle for harmonic functions, we can extract a subsequence (which we also denote by $\left.\left\{s_{k}\right\}_{k=1}^{\infty}\right)$ such that the sequence $\left\{U_{s_{k}}\right\}_{k=1}^{\infty}$ is uniformly convergent on any compact subset of the strip $\left\{z:|\operatorname{Im} z|<\frac{1}{4} H\right\}$. Let $U$ be the limiting function. Clearly, $U$ is harmonic in $\left\{z:|\operatorname{Im} z|<\frac{1}{4} H\right\}$ and $U(x)=0$ for $x \in \mathbf{R}$. Putting $s=s_{k}$ in (14) and letting $k \rightarrow \infty$, we obtain (12).

Lemma 2.2. Let $u$ be a (complex-valued) function harmonic in $\mathbf{C}_{+}$and satisfying (11). Assume that there exists a sequence $\left\{r_{k}\right\}_{k=1}^{\infty}, r_{k} \rightarrow \infty$, such that

$$
\int_{0}^{\pi}\left|u\left(r e^{i \theta}\right)\right| \sin \theta d \theta \leq \exp (o(r)), \quad r=r_{k} \rightarrow \infty .
$$

Then representation (12) holds with

$$
U(z)=k y, \quad y=\operatorname{Im} z
$$

where $k \in \mathbf{C}$ is a constant. 
Proof. We divide the proof into five parts. Without loss of generality, assume that $u$ is real-valued. Then function $U$ and measure $\nu$ in (12) are also real-valued.

Step 1. First we show that

$$
\int_{0}^{\pi}\left|U\left(r e^{i \theta}\right)\right| \sin \theta d \theta \leq \exp (o(r)), \quad r=r_{k} \rightarrow \infty .
$$

From (12) we get

$$
\begin{aligned}
\int_{0}^{\pi}\left|U\left(r e^{i \theta}\right)\right| \sin \theta d \theta \leq & \int_{0}^{\pi}\left|u\left(r e^{i \theta}\right)\right| \sin \theta d \theta \\
& +\int_{0}^{\pi}\left(\frac{r \sin \theta}{\pi} \int_{-\infty}^{\infty} \frac{d|\nu|(t)}{r^{2}+t^{2}-2 r t \cos \theta}\right) \sin \theta d \theta
\end{aligned}
$$

The first integral in the right-hand side admits the estimate (16). The change of the order of integration and simple calculations show that the second integral is $O(r)$, as $r \rightarrow \infty$. This gives (18).

Step 2. Now we show that

$$
|U(z)| \leq \exp (o(|z|)), \quad|z|=\frac{1}{2} r_{k} \rightarrow \infty
$$

where $o(|z|)$ does not depend on $\arg z$. Since $U(z)=-U(\bar{z})$, it suffices to establish (20) for $z \in \mathbf{C}_{+}$.

By the Nevanlinna formula ([3]), p. 16; [6], p. 193) we have

$$
U(z)=\frac{1}{2 \pi} \int_{0}^{\pi} \frac{\left(R^{2}-r^{2}\right) 4 R r \sin \varphi \sin \theta}{\left|R e^{i \theta}-z\right|^{2}\left|R e^{-i \theta}-z\right|^{2}} U\left(R e^{i \theta}\right) d \theta, \quad z=r e^{i \varphi}, 0<r<R, 0<\varphi<\pi .
$$

Hence

$$
|U(z)| \leq \frac{\left(R^{2}-r^{2}\right) 4 R r}{(R-r)^{4}} \frac{1}{2 \pi} \int_{0}^{\pi}\left|U\left(\operatorname{Re}^{i \theta}\right)\right| \sin \theta d \theta .
$$

Putting $R=r_{k}, r=\frac{1}{2} r_{k}$, and using (18), we get (20).

Step 3. Let us show that

$$
|U(z)|=o\left(|z|^{2}\right), \quad \text { as }|z| \rightarrow \infty,|\operatorname{Im} z|<\frac{1}{4} H .
$$

It follows from (12) that $U$ admits a representation similar to $U_{s}$ in (14) in which $u(z+i s)$ is replaced with $u(z)$ and $u(t+i s) d t$ is replaced with $d \nu(t)$. Calculations similar to those from the proof of Lemma 2.1 establish that there exists $C$ such that

$$
\int_{-\infty}^{\infty} \frac{|U(x+i y)|}{1+x^{2}} d x \leq C \text { for }|y| \leq \frac{1}{2} H
$$


and

$$
|U(z)| \leq \frac{1+\left(|z|+\frac{1}{4} H\right)^{2}}{\pi\left(\frac{1}{4} H\right)^{2}} \iint|\xi-\operatorname{Re} z|<H / 4, \frac{|U(\xi+i \eta)|}{|\eta|<H / 4} d \xi d \eta
$$

The double integral tends to 0 , as $\operatorname{Re} z \rightarrow \infty$, because its integrand is summable over the whole strip $\left\{(\xi, \eta):-\infty<\xi<\infty,|\eta|<\frac{1}{4} H\right\}$ as (23) shows. Thus, (22) is valid.

Step 4 . Let $G$ be the entire function uniquely determined by the conditions $\operatorname{Re} G(z)=U(z), G(0)=0$. We now show that:

(i) there exists a sequence $\left\{R_{k}\right\}_{k=1}^{\infty}, R_{k} \rightarrow \infty$, such that

$$
|G(z)| \leq \exp (o(|z|)) \quad \text { for }|z|=R_{k} \rightarrow \infty
$$

(ii)

$$
|G(z)|=o\left(|z|^{3}\right) \text { for }|\operatorname{Im} z| \leq \frac{1}{8} H, z \rightarrow \infty
$$

To verify (24) we use the well-known formula

$$
G^{\prime}(z)=\frac{1}{\pi \varrho} \int_{0}^{2 \pi} U\left(z+\varrho e^{i \theta}\right) e^{-i \theta} d \theta
$$

whence

$$
\left|G^{\prime}(z)\right| \leq \frac{2}{\varrho} \max _{|\zeta-z| \leq \varrho}|U(\zeta)|
$$

We get for $|z|=R_{k}=\frac{1}{2} r_{k}-\varrho$,

$$
|G(z)|=\left|\int_{0}^{z} G^{\prime}(\zeta) d \zeta\right| \leq R_{k} \max _{|\zeta| \leq R_{k}}\left|G^{\prime}(\zeta)\right| \leq R_{k} \exp \left(o\left(\frac{1}{2} r_{k}\right)\right) \leq \exp \left(o\left(R_{k}\right)\right)
$$

i.e. (24) is valid.

If $|\operatorname{Im} z| \leq \frac{1}{8} H$, it follows from (26) and (22) that $\left|G^{\prime}(z)\right|=o\left(|z|^{2}\right), z \rightarrow \infty$, whence (25) follows by integration.

Step 5. Let us complete the proof of Lemma 2.2.

Applying the well-known version of the Phragmén-Lindelöf principle for halfplane (see [7], p. 43), we conclude that (25) holds in the whole plane $\mathbf{C}$ and, by Liouville's theorem, $G$ is a polynomial of degree $\leq 2$. Since $\operatorname{Re} G(t)=U(t)=0$ for $t \in \mathbf{R}$, and $G(0)=0$, we have $G(z)=i a z^{2}+i b z, a, b \in \mathbf{R}$. Hence $U(z)=-2 a x y-b y$. If $a \neq 0$, then (23) could not be valid. Thus $U(z)=-b y$, i.e. (17) holds. 
Proof of Theorem 3. The proof of Theorem 3 differs from the proof of Lemma 2.2 by only one additional step. Namely, we should prove that, for a real-valued $u$, the estimate (18) remains valid if the condition (16) is replaced by the less restrictive condition (7).

Note that (12) implies also that the inequality (19) remains valid if we replace $|U|$ and $|u|$ with $U^{+}$and $u^{+}$, respectively. Hence,

$$
\int_{0}^{\pi} U^{+}\left(r e^{i \theta}\right) \sin \theta d \theta \leq \exp (o(r)), \quad r=r_{k} \rightarrow \infty .
$$

Setting $z=i$ in $(21)$ we get

$$
\begin{aligned}
\frac{1}{2 \pi} \int_{0}^{\pi} \frac{\left(R^{2}-1\right) 4 R \sin \theta}{\left|R e^{i \theta}-i\right|^{2}\left|R e^{-i \theta}-i\right|^{2}} \mid & U\left(R e^{i \theta}\right) \mid d \theta \\
& =\frac{2}{2 \pi} \int_{0}^{\pi} \frac{\left(R^{2}-1\right) 4 R \sin \theta}{\left|R e^{i \theta}-i\right|^{2}\left|R e^{-i \theta}-i\right|^{2}} U^{+}\left(R e^{i \theta}\right) d \theta-U(i) .
\end{aligned}
$$

Taking into account that

$$
\frac{4(R-1)^{3} \sin \theta}{(R+1)^{4}} \leq \frac{\left(R^{2}-1\right) 4 R \sin \theta}{\left|R e^{i \theta}-i\right|^{2}\left|R e^{-i \theta}-i\right|^{2}} \leq \frac{4 R^{3} \sin \theta}{(R-1)^{4}},
$$

putting $R=r_{k}$ and using (27), we obtain (18).

\section{Proof of Theorem 2}

Proof of Theorem 2. We divide the proof into four steps.

Step 1. Let us show that

$$
\log ^{+}\left|g_{j}\left(r e^{i \varphi}\right)\right| \leq \frac{C r}{\sin \varphi} \log \frac{C r}{\sin \varphi}, \quad r e^{i \varphi} \in \mathbf{C}_{+}, r \geq 1, j=1, \ldots, n,
$$

where $C$ is a positive constant.

Map $\mathbf{C}_{+}$onto $D=\{\zeta:|\zeta|<1\}$ by

$$
\zeta=\frac{z-i}{z+i}
$$

and set

$$
\tilde{h}(\zeta)=h(z), \tilde{g}_{j}(\zeta)=g_{j}(z), \quad j=1, \ldots, n
$$


Then

$$
\begin{aligned}
\tilde{h}(\zeta) & =\tilde{g}_{1}(\zeta) \ldots \tilde{g}_{n}(\zeta), \\
\tilde{g}_{n}(\zeta) & =\tilde{g}_{1}(\zeta)+\ldots+\tilde{g}_{n-1}(\zeta)
\end{aligned}
$$

Moreover, $\tilde{h} \in H^{\infty}(D)$ and $\tilde{g}_{1}, \ldots, \tilde{g}_{n-1}$ are linearly independent over C. Since the zeros of $\tilde{h}$ satisfy the Blaschke condition, equation (31) implies that the zeros of each $\tilde{g}_{j}, j=1,2, \ldots, n$, also satisfy this condition. Applying Theorem D, we get

$$
T\left(r, \frac{\tilde{g}_{j}}{\tilde{g}_{n}}\right)=O\left(\log \frac{1}{1-r}\right), \quad r \rightarrow 1, j=1, \ldots, n-1 .
$$

Using the properties of the Nevanlinna characteristic (see, e.g. [3], Chapter 1, $\S 6$; 7$]$, Chapter $6, \S 2.5)$ and taking into account that $\tilde{h} \in H^{\infty}(D)$, we obtain

$$
T\left(r, \tilde{g}_{n}\right)=\frac{1}{n} T\left(r, \frac{1}{\tilde{g}_{n}^{n}}\right)+O(1) \leq \frac{1}{n} T\left(r, \frac{\tilde{h}}{\tilde{g}_{n}^{n}}\right)+O(1),
$$

whence, using (31) and (33), we conclude

$$
\begin{aligned}
T\left(r, \tilde{g}_{n}\right) & \leq \frac{1}{n} T\left(r, \frac{\tilde{g}_{1} \ldots \tilde{g}_{n}}{\tilde{g}_{n}^{n}}\right)+O(1) \\
& \leq \frac{1}{n} \sum_{j=1}^{n-1} T\left(r, \frac{\tilde{g}_{j}}{\tilde{g}_{n}}\right)+O(1)=O\left(\log \frac{1}{1-r}\right), \quad r \rightarrow 1 .
\end{aligned}
$$

Using (33) once again, we get

$$
T\left(r, \tilde{g}_{j}\right) \leq T\left(r, \frac{\tilde{g}_{j}}{\tilde{g}_{n}}\right)+T\left(r, \tilde{g}_{n}\right)=O\left(\log \frac{1}{1-r}\right), \quad r \rightarrow 1, j=1, \ldots, n .
$$

The well-known inequality

$$
\log ^{+} M\left(r, \tilde{g}_{j}\right) \leq \frac{4}{1-r} T\left(\frac{1+r}{2}, \tilde{g}_{j}\right)
$$

where $M\left(r, \tilde{g}_{j}\right)=\max \left\{\left|\tilde{g}_{j}(\zeta)\right|:|\zeta|<r\right\}$, allows to derive from (34) that there is a positive constant $C_{1}$ such that

$$
\log ^{+}\left|\tilde{g}_{j}(\zeta)\right| \leq \frac{C_{1}}{1-|\zeta|} \log \frac{C_{1}}{1-|\zeta|}, \quad \zeta \in D, j=1, \ldots, n
$$

Using (29), we get (28). 
Step 2. Let us show that

$$
\sup \left\{\int_{-\infty}^{\infty} \frac{|\log | g_{j}(x+i y)||}{1+x^{2}} d x: 0<y<H\right\}<\infty, \quad j=1, \ldots, n
$$

where $I$ is taken from the condition (i) of Theorem 2.

We need the following simple known lemma (see, e.g. [8]).

Lemma 3.1. If a function $Q \not \equiv 0$ belongs to $H^{\infty}\left(\mathbf{C}_{+}\right)$, then for any $K>0$

$$
\sup \left\{\int_{-\infty}^{\infty} \frac{\log ^{+}|1 / Q(x+i y)|}{1+x^{2}} d x: 0<y<K\right\}<\infty
$$

To prove $(35)$, write

$$
\int_{-\infty}^{\infty} \frac{|\log | g_{j}(x+i y)||}{1+x^{2}} d x=\int_{-\infty}^{\infty} \frac{\log ^{+}\left|g_{j}(x+i y)\right|}{1+x^{2}} d x+\int_{-\infty}^{\infty} \frac{\log ^{+}\left|1 / g_{j}(x+i y)\right|}{1+x^{2}} d x
$$

The first integral in the right-hand side is bounded for $0<y<H$ by condition (i) of Theorem 2. Using condition (i) once again, we get

$$
\frac{1}{\left|g_{j}(x+i y)\right|}=\frac{1}{|h(x+i y)|} \prod_{k \neq j}\left|g_{k}(x+i y)\right| \leq \frac{C}{|h(x+i y)|}, \quad 0<y<H
$$

where $C$ is a positive constant. Hence, the boundedness of the second integral in the right-hand side of (36) is a consequence of Lemma 3.1 with $Q=h$.

Step 3. Denote by $B_{j}$ the Blaschke product formed by the zeros of $g_{j}$ and set

$$
u_{j}(z)=\log \left|\frac{g_{j}(z)}{B_{j}(z)}\right|, \quad j=1, \ldots, n .
$$

This is a harmonic function in $\mathbf{C}_{+}$. Let us show that it satisfies the assumptions of Theorem 3.

We have

$$
\int_{0}^{\pi} u_{j}^{+}\left(r e^{i \varphi}\right) \sin \varphi d \varphi \leq \int_{0}^{\pi} \log ^{+}\left|g_{j}\left(r e^{i \varphi}\right)\right| \sin \varphi d \varphi+\int_{0}^{\pi} \log ^{+}\left|\frac{1}{B_{j}\left(r e^{i \varphi}\right)}\right| \sin \varphi d \varphi .
$$

Estimate (28) implies that the first integral in the right-hand side is $O(r \log r)$, as $r \rightarrow \infty$. To estimate the second integral, we need the following lemma. 
Lemma 3.2. If a function $Q \not \equiv 0$ belongs to $H^{\infty}\left(\mathbf{C}_{+}\right)$, then

$$
\int_{0}^{\pi} \log ^{+}\left|\frac{1}{Q\left(r e^{i \varphi}\right)}\right| \sin \varphi d \varphi=O(r), \quad r \rightarrow \infty .
$$

This lemma can be easily derived from the Nevanlinna formula ([3], p. 16; [6], p. 193) or the Carleman formula ([3], p. 19; [6], p. 188), therefore we omit the proof.

Applying Lemma 3.2 to $B_{j}(z)$, we get that the second integral in (38) is $O(r)$, as $r \rightarrow \infty$. Hence, $u_{j}$ satisfies the condition (i) of Theorem 3 .

Further we have

$$
\int_{-\infty}^{\infty} \frac{\left|u_{j}(x+i y)\right|}{1+x^{2}} d x \leq \int_{-\infty}^{\infty} \frac{|\log | g_{j}(x+i y)||}{1+x^{2}} d x+\int_{-\infty}^{\infty} \frac{\log ^{+}\left|1 / B_{j}(x+i y)\right|}{1+x^{2}} d x .
$$

Using (35) for the first integral in the right-hand side and Lemma 3.1 for the second one, we see that condition (ii) of Theorem 3 is also satisfied.

Step 4. Let us now complete the proof of Theorem 2.

Applying Theorem 3, we get the representation

$$
u_{j}(z)=\frac{y}{\pi} \int_{-\infty}^{\infty} \frac{d \nu_{j}(t)}{(t-x)^{2}+y^{2}}+k_{j} y, \quad z=x+i y \in \mathbf{C}_{+},
$$

where $k_{j}$ is a real constant and $\nu_{j}$ is a real-valued Borel measure satisfying (10). Set

$$
\psi_{j}(z)=\exp \left(\frac{1}{\pi i} \int_{-\infty}^{\infty}\left(\frac{1}{t-z}-\frac{t}{1+t^{2}}\right) d \nu_{j}(t)\right) .
$$

Then, according to (37) we have

$$
g_{j}(z)=e^{i \alpha} \psi_{j}(z) B_{j}(z) e^{-i k_{j} z}, \quad \alpha \in \mathbf{R} .
$$

Let us show that $g_{j}(z) \exp \left(i k_{j} z\right)$ belongs to $H^{\infty}\left(\mathbf{C}_{+}\right)$. Clearly, this function is bounded in $\{z: 0<\operatorname{Im} z<H\}$ by condition (i) of Theorem 2. For $y \geq H$ and any fixed $N>1$ we have

$$
\begin{aligned}
\log ^{+}\left|g_{j}(z) e^{i k_{j} z}\right| & \leq \frac{y}{\pi} \int_{-\infty}^{\infty} \frac{d \nu_{j}^{+}(t)}{(x-t)^{2}+y^{2}} \\
& \leq \frac{1}{\pi H} \int_{-N}^{N} d \nu_{j}^{+}(t)+\frac{2\left(x^{2}+y^{2}\right)}{y} \int_{|t|>N} \frac{d \nu_{j}^{+}(t)}{1+t^{2}}, \quad z=x+i y .
\end{aligned}
$$

Since $N$ can be taken arbitrarily large, we get

$$
\begin{array}{ll}
\log ^{+}\left|g_{j}(z) e^{i k_{j} z}\right|=o\left(|z|^{2}\right), & |z| \rightarrow \infty, \quad \operatorname{Im} z \geq H, \\
\log ^{+}\left|g_{j}(z) e^{i k_{j} z}\right|=o(|z|), & |z| \rightarrow \infty, \quad\left|\frac{1}{2} \pi-\arg z\right|<\frac{1}{4} \pi .
\end{array}
$$

Applying the Phragmén-Lindelöf principle we conclude that (6) holds. 


\section{Proof of Theorem 1}

Theorem 1 will be proved if we prove the following fact.

Theorem 1'. Under the hypotheses of Theorem 1, the following implication holds:

$$
l\left(\mu_{1} * \mu_{2} * \ldots * \mu_{n}\right)>-\infty \Longrightarrow l\left(\mu_{j}\right)>-\infty, \quad j=1, \ldots, n
$$

Proof. Without loss of generality one may assume that $l\left(\mu_{1} * \mu_{2} * \ldots * \mu_{n}\right)=0$. The last equality implies that the Fourier transform $\hat{\mu}$ of the measure $\mu:=\mu_{1} * \ldots * \mu_{n}$ belongs to $H^{\infty}\left(\mathbf{C}_{+}\right)$.

For $z \in \mathbf{R}$, we have

$$
\begin{aligned}
\hat{\mu}(z) & =\hat{\mu}_{1}(z) \hat{\mu}_{2}(z) \ldots \hat{\mu}_{n}(z) \\
\hat{\mu}_{j}(z) & =\int_{-\infty}^{\infty} e^{i z t} d \mu_{j}(t), \quad j=1,2, \ldots, n .
\end{aligned}
$$

Condition (5) implies that the integral in the right-hand side of (42) converges absolutely and uniformly on any compact subset of $\overline{\mathbf{C}}_{+}$. Hence, $\hat{\mu}_{j}$ can be extended to $\overline{\mathbf{C}}_{+}$as a function analytic in $\mathbf{C}_{+}$and continuous in $\overline{\mathbf{C}}_{+}$. Then equation (41) holds in $\overline{\mathbf{C}}_{+}$, and, moreover, for any $H>0$ we have $\sup \left\{\left|\hat{\mu}_{j}(z)\right|: 0<\operatorname{Im} z<H\right\}<\infty$. By Theorem 2 we obtain that $\hat{\mu}_{j}(z) \exp \left(i b_{j} z\right) \in H^{\infty}\left(\mathbf{C}_{+}\right), j=1, \ldots, n$. Using the wellknown corollary of the Paley-Wiener theorem we get $l\left(\mu_{j}\right) \geq-\left|b_{j}\right|>-\infty, j=1, \ldots, n$.

It remains to prove that the condition (5) in Theorem 1 cannot be weakened by replacing 'for all' by 'there exists'. For this, we consider the measures $\mu_{1}, \mu_{2}, \mu_{3}$ defined by the Fourier transforms

$$
\hat{\mu}_{1}(z)=\frac{1}{1+i z / c}, \quad \hat{\mu}_{2}(z)=\frac{(1+i z / c)^{2}}{(1-i z / c)^{4}}, \quad \hat{\mu}_{3}(z)=\hat{\mu}_{1}(z)+\hat{\mu}_{2}(z)
$$

where $c$ is a positive constant. A direct calculation of the inverse Fourier transform shows that the condition ( 7$)$ is satisfied with the given fixed $c>0$, and $l\left(\mu_{1}\right)=l\left(\mu_{2}\right)=$ $l\left(\mu_{3}\right)=-\infty$. Nevertheless, $l\left(\mu_{1} * \mu_{2} * \mu_{3}\right)=0$.

\section{A generalization of Theorem 1}

Equality (2) is not true for arbitrary linearly independent measures $\mu_{j}$ satisfying (5). Indeed, if $n$ is even, we define $\mu_{1}$ and $\mu_{2}$ by (3) and set

$$
\mu_{2 s-1}(d x)=\mu_{1}(s d x), \quad \mu_{2 s}(d x)=\mu_{2}(s d x), \quad s=1,2, \ldots, \frac{1}{2} n .
$$


Then $\mu_{1}, \ldots, \mu_{n}$ satisfy condition (5) and $l\left(\mu_{1} * \ldots * \mu_{n}\right)=0$ while $l\left(\mu_{j}\right)=-\infty$ for all $j=1, \ldots, n$. An example in which $\mu_{2 s-1}=\mu_{1}$ and $\mu_{2 s}=\mu_{2}$, where $s=1, \ldots, \frac{1}{2} n$ and $\mu_{1}$ and $\mu_{2}$ are defined by (3), shows that Theorem $\mathrm{A}$ is also not true for arbitrary collections of linearly dependent measures satisfying (5). We now give necessary and sufficient conditions on the linear dependence of measures $\mu_{j}$ for (2) to remain true.

Let $\left\{\mu_{1}, \ldots, \mu_{n}\right\}, n \geq 2$, be a collection from measures from $M$ satisfying (5). Let $\Lambda$ be the linear span of the collection, $p=\operatorname{dim} \Lambda$. We assume that $1 \leq p \leq n-1$. One may reorder $\mu_{j}$ so that the first $p$ measures $\mu_{1}, \ldots, \mu_{p}$ form a basis of $\Lambda$. Then we have

$$
\mu_{k}=\sum_{j=1}^{p} c_{k, j} \mu_{j}, \quad p+1 \leq k \leq n,
$$

where $c_{k, j}$ 's are constants. Consider the $(n-p) \times p$ matrix

$$
C=\left(\begin{array}{cccc}
c_{p+1,1} & c_{p+1,2} & \ldots & c_{p+1, p} \\
\vdots & \vdots & \ddots & \vdots \\
c_{n, 1} & c_{n, 2} & \ldots & c_{n, p}
\end{array}\right)
$$

We say that the collection $\left\{\mu_{1}, \ldots, \mu_{n}\right\}$ is admissible if $C$ satisfies the following conditions $(\alpha)$ and $(\beta)$ :

$(\alpha)$ Each column of $C$ contains at least one non-zero element.

To introduce the second condition, observe that in some cases it is possible to delete some rows of $C$ without violating condition $(\alpha)$. Let us denote by $\widetilde{C}$ any submatrix of $C$ with the minimal number of rows that still satisfies condition $(\alpha)$. The second condition sounds as follows.

$(\beta)$ Any matrix $\widetilde{C}$ either consists of one single row, or each pair $\{\varrho, R\}$ of rows of $\widetilde{C}$ can be embedded in a set $\left\{\varrho_{1}, \ldots, \varrho_{m}\right\}$ of rows of $\widetilde{C}$, such that $\varrho_{1}=\varrho, \varrho_{m}=R$ and, for each $t, 1 \leq t \leq m-1$, $\varrho_{t}$ and $\varrho_{t+1}$ have non-zero elements in the same column (depending on $t$ ).

Observe that if $p=1$, then $C$ consists of one single column and the collection $\left\{\mu_{1}, \ldots, \mu_{n}\right\}, n \geq 2$, is always admissible. If the assumptions of Theorem 1 are satisfied, then $p=n-1$ and $C=\widetilde{C}$ consists of one single row $(1,1, \ldots, 1)$ and hence is admissible. Therefore, the following theorem can be viewed as a generalization of Theorem 1.

Theorem 4. Let $\left\{\mu_{1}, \mu_{2}, \ldots, \mu_{n}\right\}, n \geq 3$, be a collection of measures of $M$ satisfying (5). If this collection is admissible, then (2) holds.

One can check that assumptions $(\alpha)$ and $(\beta)$ hold when $\mu_{j}=\nu_{1}-\exp (2 \pi i j / n) \nu_{2}$, $j=1, \ldots, n$, where $\nu_{1}$ and $\nu_{2}$ belong to $M$ and satisfy (5). This shows that Theorem $\mathrm{C}$ follows from Theorem 4 in the same way as Theorem B follows from Theorem A. 
Note that the notion of admissibility can be extended in an evident way to collections $\left\{g_{1}, g_{2}, \ldots, g_{n}\right\}, n \geq 2$, of functions analytic and $\not \equiv 0$ in $\mathbf{C}_{+}$. Theorem 4 can be derived from the following generalization of Theorem 2 in the same way as Theorem 1 follows from Theorem 2.

Theorem 5. Let $h \neq 0$ belong to $H^{\infty}\left(\mathbf{C}_{+}\right)$. Suppose that $h=g_{1} g_{2} \ldots g_{n}$, where the functions $g_{j}, j=1,2, \ldots, n, n \geq 3$, are analytic in $\mathbf{C}_{+}$. If the collection $\left\{g_{1}, \ldots, g_{n}\right\}$ is admissible and the condition (i) of Theorem 2 is satisfied, then there exist constants $b_{j} \in \mathbf{R}$ such that (6) holds.

Proof. Observe that the condition (ii) of Theorem 2 was utilized only in the first step of its proof. If we could prove (28) only under the condition of admissibility, then the rest of the proof of Theorem 2 could be repeated. Thus we can restrict ourselves to the proof of (28). We divide it into five steps.

Step 1. As in the proof of Theorem 2, let us map $\mathbf{C}_{+}$onto $D$ by (29) and consider the functions (30). Then we again have (31) but instead of (32), we have $n-p$ equations

$$
\tilde{g}_{k}(\zeta)=\sum_{j=1}^{p} c_{k, j} \tilde{g}_{j}(\zeta), \quad k=p+1, \ldots, n
$$

where $\tilde{g}_{1}, \ldots, \tilde{g}_{p}$ form a basis of the linear span of $\left\{\tilde{g}_{1}, \ldots, \tilde{g}_{n}\right\}$. Applying Theorem D, we get

$$
T\left(r, \frac{\tilde{g}_{j}}{\tilde{g}_{k}}\right)=O\left(\log \frac{1}{1-r}\right), \quad r \rightarrow 1
$$

for $1 \leq j \leq p<k \leq n$, provided that $c_{k, j} \neq 0$.

Step 2. We show that (44) remains in force if we replace $k$ by $l, p+1 \leq l \leq n$, where $l$ is such that the $k$-th and $l$-th rows have non-zero elements in the same column (the $j_{0}$-th, say).

Indeed, since $c_{k, j_{0}} \neq 0, c_{l, j_{0}} \neq 0,(44)$ is valid for $\tilde{g}_{j_{0}} / \tilde{g}_{k}$ and $\tilde{g}_{j_{0}} / \tilde{g}_{l}$.

Whence, for any $j, 1 \leq j \leq p$,

$$
T\left(r, \frac{\tilde{g}_{j}}{\tilde{g}_{l}}\right) \leq T\left(r, \frac{\tilde{g}_{j}}{\tilde{g}_{k}}\right)+T\left(r, \frac{\tilde{g}_{j_{0}}}{\tilde{g}_{k}}\right)+T\left(r, \frac{\tilde{g}_{j_{0}}}{\tilde{g}_{l}}\right)+O(1)=O\left(\log \frac{1}{1-r}\right), \quad r \rightarrow 1 .
$$

Step 3. The next step is to show that (44) is valid for each pair $(j, k)$ where $j$ is an arbitrary integer satisfying $1 \leq j \leq p$, while $k, p+1 \leq k \leq n$, is such that the $k$-th row belongs to the submatrix $\widetilde{C}$ mentioned in the definition of admissibility. 
By the definition of $\widetilde{C}$, for any $j, 1 \leq j \leq p$, there is $l, p+1 \leq l \leq n$, such that the $l$-th row belongs to $\widetilde{C}$ and $c_{l, j} \neq 0$. Hence (44) is valid for $\tilde{g}_{j} / \tilde{g}_{l}$. Let $k$ be an arbitrary integer such that the $k$-th row belongs to $\widetilde{C}$. By condition $(\beta)$ of admissibility, there is a set $\left\{l_{1}, \ldots, l_{m}\right\}$ of integers, $l_{1}=l, l_{m}=k$, such that the $l_{t}$-th row belongs to $\widetilde{C}$ and the $l_{t}$-th and $l_{t+1}$-th rows, $1 \leq t \leq m-1$, have non-zero elements in the same column. As was shown in Step 2, (44) remains in force for $\tilde{g}_{l_{1}} / \tilde{g}_{l_{2}}$. Repeating this procedure $m$ times, we show that $(44)$ is valid for $\tilde{g}_{l_{1}} / \tilde{g}_{l_{m}}$.

Step 4. Now we show that (44) remains in force for $\tilde{g}_{j} / \tilde{g}_{k}$ with any $k$ and $j$ such that $1 \leq k, j \leq p$.

Indeed, according to Step 3 we have (44) for $\tilde{g}_{k} / \tilde{g}_{l}$ with any $k$ and $j$ such that $1 \leq k, j \leq p$ and for $\tilde{g}_{j} / \tilde{g}_{l}$ with any $l$ such that the $l$-th row belongs to $\widetilde{C}$. Hence

$$
T\left(r, \frac{\tilde{g}_{k}}{\tilde{g}_{j}}\right) \leq T\left(r, \frac{\tilde{g}_{k}}{\tilde{g}_{l}}\right)+T\left(r, \frac{\tilde{g}_{l}}{\tilde{g}_{j}}\right)=O\left(\log \frac{1}{1-r}\right), \quad r \rightarrow 1 .
$$

Step 5. Let us complete the proof. To this end, we show that

$$
T\left(r, \tilde{g}_{j}\right)=O\left(\log \frac{1}{1-r}\right), \quad r \rightarrow 1,1 \leq j \leq n .
$$

First we consider the case $1 \leq j \leq p$. In this case

$$
T\left(r, \tilde{g}_{j}\right) \leq \frac{1}{n} \sum_{k=1}^{n} T\left(r, \frac{\tilde{g}_{k}}{\tilde{g}_{j}}\right)+O(1)=\frac{1}{n}\left(\sum_{k=1}^{p} T\left(r, \frac{\tilde{g}_{k}}{\tilde{g}_{j}}\right)+\sum_{k=p+1}^{n} T\left(r, \frac{\tilde{g}_{k}}{\tilde{g}_{j}}\right)\right)+O(1) .
$$

Clearly,

$$
\sum_{k=1}^{p}=O\left(\log \frac{1}{1-r}\right), \quad r \rightarrow 1
$$

in virtue of Step 4 . If $p+1 \leq k \leq n$, then, using (43) and properties of the characteristic $T$, we have

$$
T\left(r, \frac{\tilde{g}_{k}}{\tilde{g}_{j}}\right) \leq \sum_{s=1}^{p} T\left(r, \frac{\tilde{g}_{s}}{\tilde{g}_{j}}\right)+O(1)=O\left(\log \frac{1}{1-r}\right), \quad r \rightarrow 1
$$

Hence

$$
\sum_{k=p+1}^{n}=O\left(\log \frac{1}{1-r}\right), \quad r \rightarrow 1
$$

and (45) follows for $1 \leq j \leq p$. Using this, we have for $p+1 \leq j \leq n$,

$$
T\left(r, \tilde{g}_{j}\right)=T\left(r, \sum_{s=1}^{p} c_{j, s} \tilde{g}_{s}\right) \leq \sum_{s=1}^{p} T\left(r, \tilde{g}_{s}\right)+O(1)=O\left(\log \frac{1}{1-r}\right) .
$$

The estimate (28) follows from this in the same way as in the proof of Theorem 2 . 
We conclude the paper by the remark that the condition of admissibility in Theorem 5 cannot be weakened. Namely, if an $(n-p) \times p$ matrix $C, 2 \leq p \leq n$, does not satisfy at least one of the conditions $(\alpha)$ and $(\beta)$, then it is possible to define a collection $\left\{\mu_{1}, \ldots, \mu_{n}\right\}$ having $C$ as the corresponding matrix and such that (5) is satisfied, but (2) does not hold. We will only confirm this by a typical example, since in the general case one needs to introduce a somewhat complicated notation.

Example. Consider the case $n=6, p=4$,

$$
C=\left(\begin{array}{llll}
1 & 1 & 0 & 0 \\
0 & 0 & 1 & 1
\end{array}\right)
$$

Define

$$
\begin{array}{ll}
\hat{\mu}_{1}(z)=\left(1+\hat{\nu}_{1}(z)\right) e^{\cos z}, & \hat{\mu}_{2}(z)=\left(1+\hat{\nu}_{2}(z)\right) e^{\cos z}, \\
\hat{\mu}_{3}(z)=\left(1+\hat{\nu}_{3}(z)\right) e^{-\cos z}, & \hat{\mu}_{4}(z)=\left(1+\hat{\nu}_{4}(z)\right) e^{-\cos z}, \\
\hat{\mu}_{5}(z)=\hat{\mu}_{1}(z)+\hat{\mu}_{2}(z), & \hat{\mu}_{6}(z)=\hat{\mu}_{3}(z)+\hat{\mu}_{4}(z),
\end{array}
$$

where $\nu_{j}$ are measures, linearly independent over $\mathbf{C}$, such that $l\left(\nu_{j}\right)>0, j=1,2,3,4$. Clearly, all measures satisfy the condition (5) but (2) does not hold.

Acknowledgment. The authors thank Professor Yngve Domar whose remarks to [8] given in a letter to the second named author were used in the proof of Theorem 3. We are also indebted to Professor Mikhail Sodin for several useful remarks.

The research of the second and third named authors is partially supported by INTAS Grant No. 96-0858. The research was done during a visit of the third named author to Bilkent University at Ankara. This visit was supported by the Scientific and Technical Research Council of Turkey (TÜBİTAK).

\section{References}

1. Cartan, H., Sur les zéros des combinaisons linèares de $p$ functions holomorphes donnèes, Mathematica (Cluj) 7 (1933), 5-31.

2. DOMAR, Y., Extension of the Titchmarsh convolution theorem with applications in the theory of invariant subspaces, Proc. London. Math. Soc. 46 (1983), 288300.

3. Gol'Dberg, A. and Ostrovski, I. V., The Value Distribution of Meromorphic Functions, Nauka, Moscow, 1970 (Russian).

4. Koosis, P., Introduction to $H_{p}$ Spaces, 2nd ed., Cambridge Univ. Press, Cambridge, 1998.

5. Levin, B. Ya, Distribution of Zeros of Entire Functions, Amer. Math. Soc., Providence, R. I., 1980.

6. Levin, B. YA., Lectures on Entire Functions, Amer. Math. Soc., Providence, R. I., 1996.

7. Nevanlinna, R., Eindeutige analytische Funktionen, 2nd ed., Springer-Verlag, Berlin, 1953. English transl.: Analytic Functions, Springer-Verlag, Berlin, 1970. 
8. Ostrovskil, I. V., Generalization of the Titchmarsh convolution theorem and the complex-valued measures uniquely determined by their restriction to a halfline, in Stability Problems for Stochastic Models (Zolotarev, V. M. and Kalashnikov, V. V., eds.), Lecture Notes in Math. 1155, pp. 256-283, SpringerVerlag, Berlin-Heidelberg, 1985.

Received May 25, 2000

\author{
Seçil Gergün \\ Department of Mathematics \\ Bilkent University \\ 06533 Bilkent \\ Ankara \\ Turkey \\ Iossif V. Ostrovskii \\ Department of Mathematics \\ Bilkent University \\ 06533 Bilkent \\ Ankara \\ Turkey \\ and \\ B. Verkin Institute for \\ Low Temperature Physics and Engineering \\ 61164 Kharkiv \\ Ukraine
}

\author{
Alexander Ulanovskii \\ Høgskolen i Stavanger \\ P.O. Box 2557 \\ Ullandhaug \\ NO-4004 Stavanger \\ Norway
}

